\title{
Efecto de la edad al primer parto y los días abiertos en un bovino doble propósito sobre la huella hídrica y de carbono
}

\section{Effect of the age at first calving and open days in a bovine dual purpose on the water and carbon footprints}

\author{
Efeito da idade ao primeiro parto e os dias abertos \\ em um bovino de duplo propósito sobre a impressão \\ hídrica e de carbono
}

\section{Raúl Andrés Molina Benavides', Hugo Sánchez Guerrero², Jose Reinel Uribe Ceballos ${ }^{3}$ \& Alberto Stanislao Atzori ${ }^{4}$}

\begin{abstract}
1Zootecnista, Magister en Ciencias Agrarias, Doctor en Ciencias Agrarias. ${ }^{2}$ Zootecnista, Especialista en Producción de Ganado Lechero, Magister en Producción Animal. ${ }^{3}$ Ingeniero de Sistemas, Especialista en Administración Énfasis en Finanzas, Magister en Administración, ${ }^{4} \mathrm{PhD}$ in Animal Science.
\end{abstract}

1Departamento de Ciencia Animal. ²Departamento de Ciencias Básicas. 1,2Universidad Nacional de Colombia Sede Palmira. Colombia. ${ }^{3}$ Dipartimento di Agraria, University of Sassari, Sassaari 07100, Italy.

1,2,3ramolinab@unal.edu.co, ${ }^{4}$ asatzori@uniss.it

\section{Resumen}

La actividad ganadera contribuye con la emisión de gases de efecto invernadero (EGEI) y el consumo de agua fresca (CA). Por ende, el objetivo de esta investigación fue estimar el efecto de la edad al primer parto (EPP) y los días abiertos (DA) en un bovino doble propósito en pastoreo, trópico bajo colombiano, sobre las EGEl y el CA. Esta estimación se logró integrando 4 metodologías existentes: Dinámica de Sistemas, Large Ruminal Nutrition System, Huella de carbono y Huella hídrica. Durante toda su vida, 91 meses, desde el nacimiento hasta el sacrificio, la vaca produjo $5.879 \mathrm{~kg}$ de leche -4 lactancias-, emitió $16.066 \mathrm{~kg} \mathrm{CO}$ eq y consumió $12.804 .569 \mathrm{~L}$ de agua. Al disminuir la EPP en un mes, el CA y las EGEl se redujeron en $123.156 \mathrm{~L}$ y $155 \mathrm{~kg} \mathrm{CO}$ eq, respectivamente. Cuando se mermaron los DA en 21, las EGEI bajaron $647 \mathrm{~kg} \mathrm{CO}$ eq y el CA se redujo en 455.455
L. Parámetros reproductivos como la EPP y los DA tienen una fuerte influencia sobre la dinámica del animal en el hato, afectando su productividad, emisiones de gases de efecto invernadero y consumo de agua.

Palabras clave: agua, ganadería, gases de efecto invernadero, modelos.

\section{Abstract}

Livestock activity contributes to the emission of greenhouse gases (EGHG) and consumption of fresh water (CW). Therefore, the objective of this study was to estimate the effect of age at first calving (AFC) and open days (OD) in a dual-purpose cattle grazing, under Colombian tropics conditions, on EGHG and CW. This estimate was achieved using the integration of four existing methodologies: 
Dynamic System, Large Ruminal Nutrition System, Carbon Footprint and Water Footprint. It was found that during all his life (91 months, from birth to slaughter), cow produced $5.879 \mathrm{~kg}$ of milk (four lactations), released $16.066 \mathrm{~kg}$ of $\mathrm{CO}_{2}$ eq and consumed 12.804.569 liters of water. The decrease in a month of $A F C$, the $C W$ and EGHG reduced in $123.156 \mathrm{~L}$ and $155 \mathrm{~kg} \mathrm{CO} 2$ eq, respectively. When the open days reduced in 21 days, EGHG reduced 647 $\mathrm{kg} \mathrm{CO}$ eq and CW decreced in 455.455 I. Reproductive parameters such as AFC and OD, have a strong influence on the dynamics of the animal in the herd, affecting her productivity, emissions of greenhouse gases and water consumption.

Key words: Greenhouse gases, livestock, models, water.

\section{Resumo}

A atividade ganadeira contribui com a emissão de gases efeito estufa (EGEI) e o consumo de agua fresca
(CA). Assim, o objetivo desta pesquisa foi estimar o efeito da idade ao primeiro parto (EPP) e os dias abertos (DA) em um bovino de duplo propósito em pastoreio, trópico baixo colombiano, sobre EGEI e CA. Essa estimação foi obtida integrando 4 metodologias já existentes: Dinâmica de Sistemas, Large Ruminal Nutrition System, Impressão de carbono e Impressão hídrica. Durante a vida toda, 91 meses, desde o nascimento até o sacrifício, a vaca produz $5.879 \mathrm{~kg}$ de leite -4 em lactação-, emitiu 16.066 kg CO${ }_{2}$ eq e consumiu 12.804.569 L de água. Ao diminuir a EPP em um mês, o CA e EGEl foram reduzidos em $123.156 \mathrm{~L}$ e $155 \mathrm{~kg}$ $\mathrm{CO}_{2} \mathrm{eq}$, respectivamente. Quando diminuíram os $\mathrm{DA}$ em 21, os EGEl diminuíram $647 \mathrm{~kg} \mathrm{CO} \mathrm{CO}_{2}$ eq o CA foi reduzido em $455.455 \mathrm{~L}$. Parâmetros reprodutores como EPP e os DA tem forte influência sobre a dinâmica do animal, afetando sua produtividade, emissões de gases efeito estufa e consumo de água.

Palavras-chave: água, ganadeira, gases de efeito estufa, modelos.

\section{Introducción}

Todos los seres humanos para su conservación y bienestar, requieren directa o indirectamente de los recursos naturales (NAP, 2011). La interacción entre los animales domésticos y su ambiente, modulados por el manejo del hombre, es lo que conocemos hoy como sistemas ganaderos. De estos sistemas, se derivan productos -leche, carne, huevos, entre otros- que son esenciales para la vida humana, sin embargo, sus procesos de elaboración pueden causar efectos ambientales. Por ejemplo, el sector ganadero ha sido identificado como un fuerte consumidor de agua fresca en el mundo (IDF, 2010; Mekonnen \& Hoekstra, 2012) sobreexplotando este bien en muchas partes del planeta. Emplea más del 8\% del agua usada globalmente, la mayor porción de ésta es utilizada para la irrigación de cultivos para alimentación del ganado. Toda el agua destinada para los procesos de producción, bebida y servicios es insignificante a nivel global, menos del 1\% del agua global, pero esto puede ser importante en áreas secas, en términos de proporción de agua usada (Schlink, et al., 2010). La cantidad de agua consumida depende de las condiciones de manejo de los predios, del tipo de suelo, tipo de cultivo y de las condiciones climáticas imperantes (Broussain, 2011).

De igual manera, la actividad ganadera contribuye a la generación de gases de efecto invernadero (GEI). La modificación en la composición de estos gases en la atmósfera ha conducido a alteraciones climáticas con relación a su comportamiento histórico, hecho conocido como Cambio Climático (Key et al., 2014). La FAO estima que el sector ganadero contribuye con $7.1 \mathrm{Gt}$ de $\mathrm{CO}_{2}$ eq por año, las cuales representan el $14,5 \%$ del total de emisiones antrópicas. De este total, el ganado de carne es responsable del $41 \%$, el ganado de leche del $20 \%$, cerdos $9 \%$, pollos y huevos $8 \%$ (Gerber et al., 2013). Actualmente, la actividad ganadera practicada en el trópico ha 
cobrado alto interés en la comunidad científica por tres razones: la primera es debido al incremento en el consumo de proteína de origen animal de los países en desarrollo (FAO, 2009); la segunda, es la alta contribución a las emisiones de gases de efecto invernadero (Gerber et al., 2011; Herrero et al., 2011) y la tercera, es que muchos de los ganaderos en el trópico son pobres, por lo cual, mejorando la actividad ganadera, se mitiga la pobreza en estos países (World Bank, 2009; Herrero et al., 2013).

En América Latina tropical, la actividad ganadera representa el uso más importante de la tierra, siendo la actividad que más contribuye al producto interno agropecuario de los diferentes países (Barragan, Mahecha \& Cajas, 2015). Colombia posee un hato bovino cercano a 23 millones de cabezas ubicados en 39.2 millones de ha, presentando una carga de 0.6 animales/ha. Esta cifra no ha variado significativamente en los últimos veinte años, lo que revela la pobre transformación tecnológica del sector ganadero (PNUD, 2011). De este inventario total, el 56\% son hembras, $26 \%$ son machos y el restante son animales menores de un año. Solamente el $2 \%$ de este total es clasificado como lechería especializada, el $38 \%$ se dedica al doble propósito y el $60 \%$ restante se ocupa de las actividades de ceba y cría en forma extensiva (Fedegan, 2013).

Debido a la importancia socioeconómica del sector ganadero para el país, ya que contribuye con el $45 \%$ de la producción agropecuaria, genera el $7 \%$ del total de empleos, produce el 1,6\% de la riqueza nacional, participa con el $20 \%$ del PIB agropecuario y representa el 53\% del PIB pecuario (DANE, 2011; Fedegan, 2013), se debe iniciar una transformación significativa de este sector, que responda a los retos que lo vinculan con la seguridad alimentaria, crecimiento económico, cambio climático y un muy posible y cercano escenario de posconflicto (Palmett, 2015, Perfetti et al., 2013). Aunque existen practicas de mitigación aplicables para los diferentes sistemas de producción mundial que pueden mejorar la productividad animal y su eficiencia reproductiva, conllevando a reducciones en el uso de recursos naturales y disminución en las emisiones de GEl; no están muy bien documentadas y respaldadas por información completa, consolidada y científica (Hristov et al., 2013). Este hecho motivó a plantear como objetivo de este trabajo, conocer el efecto de parámetros zootécnicos, como la edad al primer parto y los días abiertos en una hembra bovina doble propósito, sobre la cantidad de agua utilizada $(L)$ y gases de efecto invernadero emitidos ( $\mathrm{kg} \mathrm{CO}_{2}$ eq) por $\mathrm{kg}$ de producto generado (kg leche).

\section{Metodología}

Se simuló el comportamiento de una hembra bovina doble propósito (Gyr x Holstein) desde su nacimiento hasta el sacrificio, con el objetivo de conocer las cantidades de agua consumida (L) y los GEI ( $\mathrm{CO}_{2}$ eq) emitidos durante toda su vida. Su sacrificio se realizó un mes después de cumplir la cuarta lactancia, promedio nacional documentado por Fedegan (2012). El animal estaba bajo condiciones de pastoreo en el trópico bajo Colombiano. La principal fortaleza de este trabajo fue la integración de 4 metodologías existentes: Large Ruminal Nutrition System (LRNS), huella hídrica (Chapagain \& Hoekstra, 2003), huella de carbono (Rotz, Montes \& Chianese, 2010) y dinámica de sistemas (Sterman, 2000).

El modelo matemático nutricional LRNS, se usó para estimar el consumo de materia seca (MS) diario en cada una de las etapas fisiológicas del animal, en este caso el consumo del forraje pastoreado, de acuerdo a las características del animal (peso vivo, edad), del forraje (FDN, PC, MS, Energía, EE) y el clima (temperatura ambiental, humedad relativa). También permitió conocer la producción de leche diaria cuando el animal se encontraba lactando. Las emisiones de GEl se estimaron usando las ecuaciones nivel 2 del Panel Intergubernamental del Cambio Climático (IPCC, del inglés Intergovernmental Panel on Climate Change), con las cuales se obtuvieron las cantidades de metano entérico, metano de las excretas y óxido nitroso (IPCC, 2006 a, b). Estimados los $\mathrm{kg}$ producidos de estos gases, se convirtieron a $\mathrm{kg}$ de $\mathrm{CO}_{2}$ eq usando las ecuaciones 1, 2 y 3 (IPCC, 2007): 
$1 \mathrm{~kg}$ de $\mathrm{CO}_{2}=1 \mathrm{~kg}$ de $\mathrm{CO}_{2}$ eq;

$1 \mathrm{~kg}$ de $\mathrm{CH}_{4}=25 \mathrm{~kg}$ de $\mathrm{CO}_{2}$ eq;

$1 \mathrm{~kg}$ de $\mathrm{N}_{2} \mathrm{O}=298 \mathrm{~kg}$ de $\mathrm{CO}_{2}$ eq.

Obtenidos los $\mathrm{kg}$ de $\mathrm{CO}_{2}$ eq totales, emitidos durante toda la vida del animal, se dividieron entre la leche producida durante las lactancias que permaneció en el hato, con el fin de hallar la huella de carbono ( $\mathrm{kg} \mathrm{CO}$ eq / kg leche).

La cantidad de agua utilizada por el animal, se calculó para los tres componentes que conforman la huella hídrica: bebida, servicios y alimento. El agua para la bebida del animal se estimó usando las ecuaciones 4,5 y 6 .

Consumo total agua (vaca lactante) (L/vaca/día) $=$ $\left(-26.12+\left(1.516{ }^{*}\right.\right.$ Temperatura media $\left({ }^{\circ} \mathrm{C}\right)+(1.299$

* Producción promedia de leche $(\mathrm{kg} / \mathrm{vaca} / \mathrm{día}))$ $+(0.058$ * Peso promedio vacas lactantes $(\mathrm{kg}))+$ (0.406 * Na ingerido)) (Meyer et al. ,2004)

Consumo total agua (vaca seca) ( $/$ vaca/día) $=$ $(11.34+(4.63$ * Consumo MS (kg/vaca/día)) - (0.036 * 330$)+\left(0.84\right.$ * Temperatura media $\left.\left.\left({ }^{\circ} \mathrm{C}\right)\right)\right)($ Stockdale \& King, 1983)

Consumo total agua (otras categorías) (I/animal/día) = Consumo MS (kg/animal/día) * $(3.413+0.01595$ * $\operatorname{EXP}\left(0.01595\right.$ * Temperatura media $\left.\left.\left({ }^{\circ} \mathrm{C}\right)\right)\right)$ (Winchester \& Morris, 1956)

Las cantidades de agua usada para los servicios del animal (lavado de establos, tinas, pezones, entre otras) se tomaron del documento de Chapagain \& Hoekstra (2003). En este, las terneras no gastaban agua, las novillas de vientre consumían $4 \mathrm{~L}$ /día y las vacas usaban $5 \mathrm{~L}$ diarios. El agua empleada para la producción del alimento, forraje, estaba en función de la evapotranspiración del cultivo, pasto, la producción de forraje en el sistema y la cantidad de forraje verde consumido por los animales del hato. La evapotranspiración se estimó usando el método de Thorntwaite (1948).

Obtenidos los $L$ totales de agua consumida durante toda la vida del animal, se dividieron entre la leche producida durante las lactancias que permaneció en el hato, con el fin de hallar la huella hídrica ( $\mathrm{L} \mathrm{H}_{2} \mathrm{O} / \mathrm{L}$ leche).

La evolución del animal fue simulada usando la metodología de dinámica de sistemas (DS). El modelo se construyó usando los principales componentes de esta disciplina como son los niveles, flujos, retroalimentación y retrasos (Sterman, 2000). Los niveles son acumulaciones dentro del sistema, los flujos son los únicos que modifican los niveles, la retroalimentación representa la cadena de causalidad dentro del sistema y los retrasos determinan el tiempo de permanencia en los niveles, ocasionando inestabilidad y oscilación (Guimaraes, Tedeschi \& Teixeira, 2009).

El modelo fue construido con la ayuda del software Vensim PLE Plus (Ventana System, Inc.), este estuvo conformado por 10 niveles, los cuales representaban las diferentes etapas fisiológicas por las cuales pasaba el animal durante su vida (Figura 1). Posterior a su construcción, los valores estimados de materia seca ingerida, producción de leche, agua utilizada y GEI emitidos para cada categoría, se adicionaron al modelo como variables externas. 


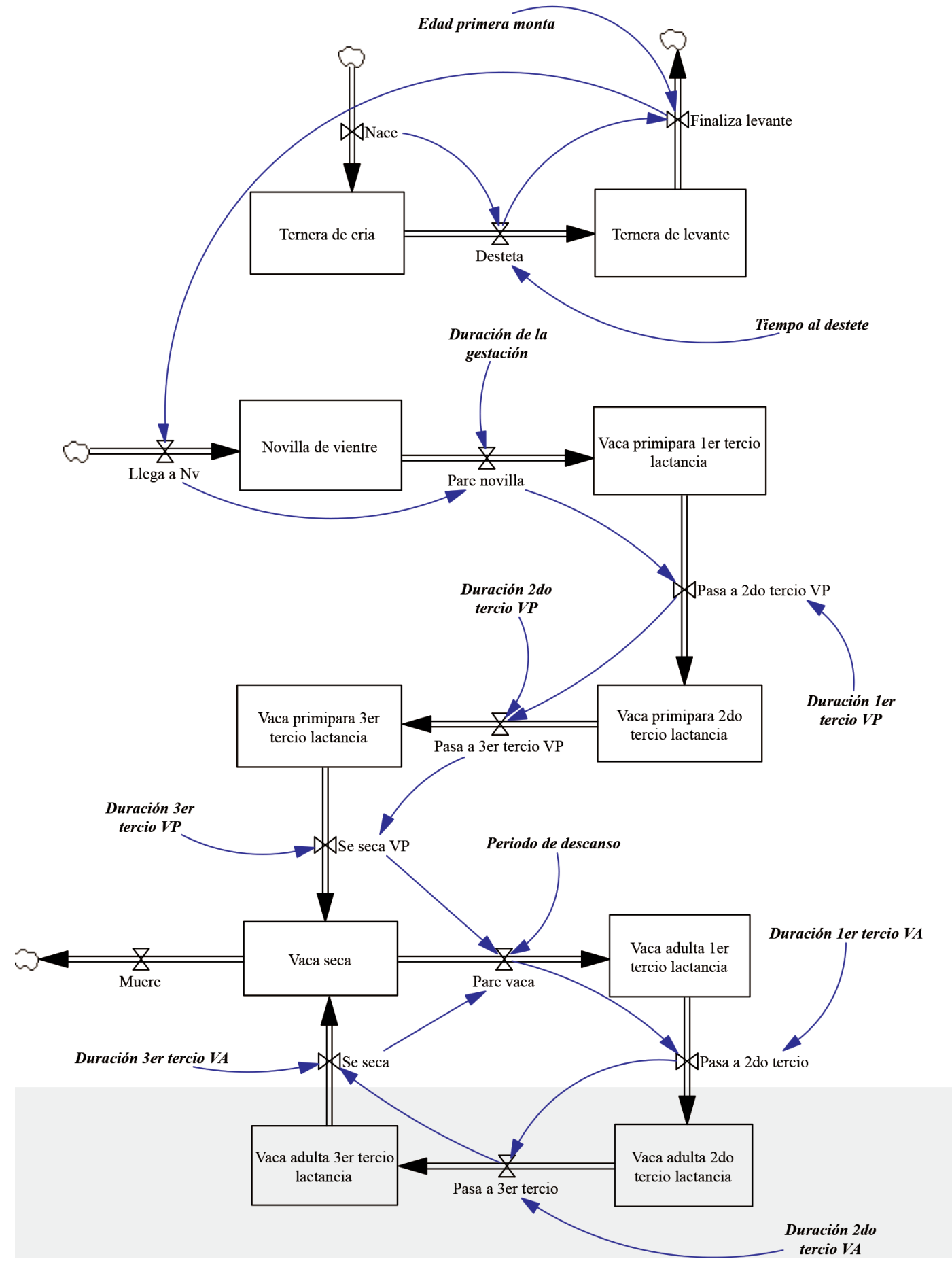

Figura 1. Modelo mediante DS representando las etapas fisiológicas por las que cursa un bovino doble propósito (hembra) durante toda su vida. Las variables en cursiva determinan los tiempos de permanencia en cada nivel. Nv: Novilla de vientre, VP: Vaca primípara, VA: Vaca adulta. 
En la Tabla 1, se muestran las principales variables utilizadas para la estimación de los consumos de materia seca, producción de leche, GEI, consumo de agua y construcción del modelo DS.

Tabla 1. Variables utilizadas para la construcción del modelo DS, consumo de materia seca, huellas de carbono e hídrica con sus respectivos valores iniciales y fuentes de referencia.

\begin{tabular}{|c|c|c|}
\hline Parámetro & Valor & Fuente \\
\hline \multicolumn{3}{|c|}{ Animal } \\
\hline Edad al destete & 9 meses & Fedegan 2012 \\
\hline Edad primera monta & 27 meses & Fedegan 2012 \\
\hline Intervalo entre partos & 456 días & Fedegan 2012 \\
\hline Duración lactancia & 9 meses & Fedegan 2012 \\
\hline Periodo de descanso & 171 días & Fedegan 2012 \\
\hline Peso vivo vaca adulta & $470 \mathrm{~kg}$ & Fedegan 2012 \\
\hline Peso al destete & $150 \mathrm{~kg}$ & Fedegan 2012 \\
\hline Peso al nacimiento & $35 \mathrm{~kg}$ & Fedegan 2012 \\
\hline \multicolumn{3}{|c|}{ Forraje } \\
\hline FDN & $67 \%$ & Peters et al., 2011 \\
\hline Proteína cruda & $10 \%$ & Peters et al., 2011 \\
\hline Extracto etéreo & $1,6 \%$ & Peters et al., 2011 \\
\hline Materia seca & $25 \%$ & Peters et al., 2011 \\
\hline Energía & $16,07 \mathrm{MJ} / \mathrm{kg}$ & Peters et al., 2011 \\
\hline kg forraje verde & $0,7 \mathrm{~kg} / \mathrm{m}^{2}$ & Molina, 2015 \\
\hline \multicolumn{3}{|c|}{ Clima } \\
\hline Temperatura media & $23^{\circ} \mathrm{C}$ & www.ideam.gov.co \\
\hline Humedad relativa & $75 \%$ & www.ideam.gov.co \\
\hline
\end{tabular}

Una de las ventajas de trabajar con modelos de simulación, es observar la reacción del sistema a una medida tomada. De esta manera se establecieron 2 políticas técnicas: i) reducción en la edad al primer parto (EPP), ii) reducción en los días abiertos (DA), con el fin de analizar la respuesta a dichas alteraciones. Las políticas se plantearon de acuerdo a las recomendaciones propuestas en los documentos de Pereira (2007); Lascano, et al (2009) y Molina (2015). También siguiendo la recomendación hecha por Ray Stata, en La Quinta disciplina (Senge, 1990), en donde propone como estrategia para mejorar el desempeño del sistema, minimizar las demoras del mismo. Para la política 1, se redujo gradualmente el valor de la EPP hasta un valor biológicamente posible para condiciones tropicales, desde el valor base de 36 meses, reportado como valor promedio nacional, hasta 30 meses. Para la política 2, se redujo progresivamente en 21 días, ciclo estral del bovino, el tiempo entre el parto y la concepción, iniciando desde el valor base de 171 días, reportado como valor promedio nacional, hasta 66 días.

\section{Resultados}

Los resultados de emisiones de gases de efecto invernadero y huella de carbono obtenidos, fueron presentados en el XIII congreso latinoamericano y encuentro colombiano de dinámica de sistemas (Molina, Sánchez \& Atzori, 2015). 
El LRNS permitió estimar el consumo de materia seca (kg/día) para cada una de las etapas del animal, al igual que la producción de leche en sus diferentes lactancias.

En la tabla 2, se muestran los resultados obtenidos:

Tabla 2. Consumo de materia seca (MS) y producción de leche en cada una de las etapas del animal

\begin{tabular}{l|c|c}
\hline Etapa & Consumo MS (kg/día) & Producción de leche (kg/día) \\
\hline Ternera de Cría & $2,2-3,6$ & 0 \\
\hline Ternera de levante & $4,1-8,7$ & 0 \\
\hline Novilla de vientre & $9,1-10,2$ & 5,3 \\
\hline Vaca prim. 1er TL* & 10,5 & 5,4 \\
\hline Vaca prim. 2do TL & 10,6 & 5,1 \\
\hline Vaca prim. 3er TL & 10,8 & 5,5 \\
\hline Vaca adulta 1er TL & 10,9 & 5,5 \\
\hline Vaca adulta 2do TL & 10,9 & 5,2 \\
\hline Vaca adulta 3er TL & 10,9 & 0 \\
\hline Vaca seca & 11 & \\
\hline
\end{tabular}

${ }^{*}$ Prim= primípara; $T L=$ Tercio de Lactancia.

Las cantidades de leche producida diariamente, se encuentran en el rango de los datos presentados por Fedegan (2012), en donde los animales doble propósito pueden producir entre 3,5 L (promedio nacional) y $6,8 \mathrm{~L}$ (fincas sobresalientes). Con los datos obtenidos mediante el LRNS, más la información composicional del alimento consumido por los animales, se estimaron las cantidades de gases de efecto invernadero $\left(\mathrm{CH}_{4}\right.$ y $\left.\mathrm{N}_{2} \mathrm{O}\right)$ emitidas por el animal a través de sus diferentes etapas de vida. En la Tabla 3, se muestran los resultados obtenidos utilizando las ecuaciones del IPCC (2006 a, b).

Tabla 3. Emisiones mensuales de $\mathrm{CH}_{4}$ y $\mathrm{N}_{2} \mathrm{O}$ en cada una de las etapas del animal

\begin{tabular}{l|c|c|c}
\hline Etapa & CH4 entérico (kg/mes) & CH4 excretas (kg/mes) & N2O (kg/mes) \\
\hline Ternera de cría & 1,66 & 0,04 & 0,04 \\
\hline Ternera de levante & $2,92-4,77$ & $0,07-1,2$ & $0,07-1,1$ \\
\hline Novilla de vientre & 5,54 & 0,14 & 0,12 \\
\hline Vaca prim. 1er TL & 6,15 & 0,2 & 0,14 \\
\hline Vaca prim. 2do TL & 6,15 & 0,2 & 0,14 \\
\hline Vaca prim. 3er TL & 6,15 & 0,2 & 0,14 \\
\hline Vaca adulta 1er TL & 6,72 & 0,17 & 0,15 \\
\hline Vaca adulta 2doTL & 6,72 & 0,17 & 0,15 \\
\hline Vaca adulta 3er TL & 6,72 & 0,17 & 0,15 \\
\hline Vaca seca & 6,67 & 0,17 & 0,15 \\
\hline
\end{tabular}


El agua utilizada por el animal durante toda su vida, para cada una de sus etapas, se exhibe en la Tabla 4. Los resultados se presentan para los tres componentes de la huella hídrica (bebida, servicios, alimento) (Chapagain \& Hoekstra, 2003).

Tabla 4. Agua mensual utilizada (bebida, servicios, alimento) en cada una de las etapas del animal.

\begin{tabular}{l|c|c|c}
\hline Etapa & Agua bebida (L/mes) & Agua servicios (L/mes) & Agua alimento (L/mes) \\
\hline Ternera de cría & $51-243$ & 0 & $1.515-54.554$ \\
\hline Ternera de levante & $487-791$ & 122 & $57.951-131.841$ \\
\hline Novilla de vientre & 1.156 & 122 & 145.480 \\
\hline Vaca prim. 1er TL & 2.252 & 152 & 159.118 \\
\hline Vaca prim. 2do TL & 2.252 & 152 & 160.634 \\
\hline Vaca prim. 3er TL & 2.252 & 152 & 163.665 \\
\hline Vaca adulta 1er TL & 2.252 & 152 & 165.180 \\
\hline Vaca adulta 2doTL & 2.252 & 152 & 165.180 \\
\hline Vaca adulta 3er TL & 2.252 & 152 & 165.180 \\
\hline Vaca seca & 2.099 & 152 & 166.696 \\
\hline
\end{tabular}

*prim= primípara; $\mathrm{TL}=$ Tercio de Lactancia

\section{Línea Base}

De acuerdo a los parámetros zootécnicos introducidos en el modelo DS, el animal presentó cuatro lactancias en el transcurso de 91 meses (Figura 2). La primera con una producción de $1.442 \mathrm{~kg}$ de leche, las tres siguientes con producciones de 1.479 kg. Estas cantidades de leche estimadas, concuerdan con los datos reportados por Fedegan (2012), en donde animales doble propósito pueden producir entre 1.200 y $1.600 \mathrm{~kg}$ de leche por lactancia. En total, la producción de leche durante su vida útil fue de $5.879 \mathrm{~kg}$.

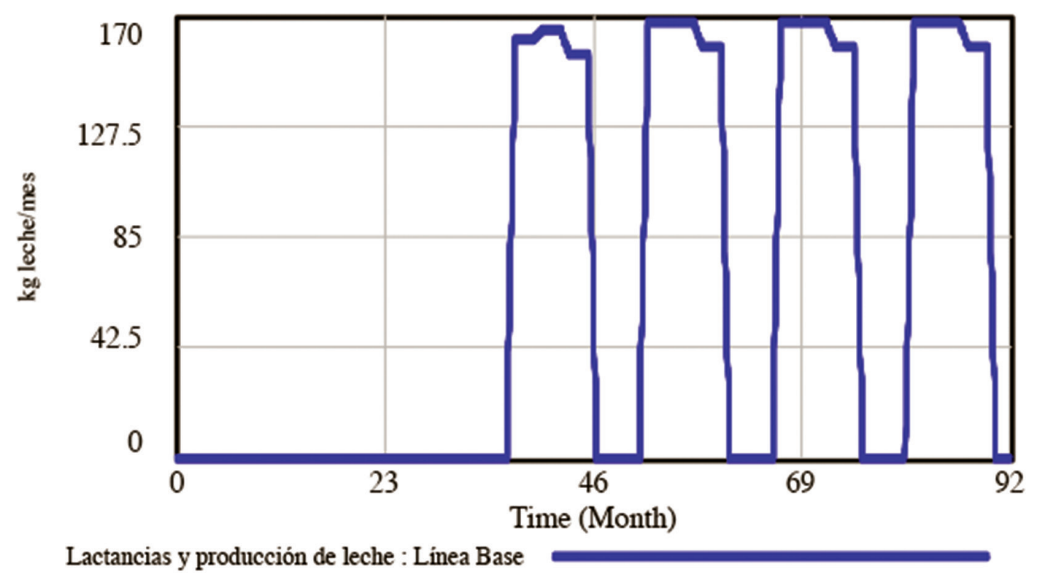

Figura 2. Número de lactancias y cantidad de leche $(\mathrm{kg})$ producida durante 92 meses de vida para un animal doble propósito. 
Por otro lado, las emisiones de GEI y consumo de agua durante toda su vida fueron de $16.066 \mathrm{~kg}$ $\mathrm{CO}_{2}$ eq y $12.804 .569 \mathrm{~L}$, respectivamente. Al dividir estas cantidades entre la leche total producida, se encontró que el animal necesitaba $2.178 \mathrm{~L}$ de agua y emitía $2,73 \mathrm{~kg} \mathrm{CO}$ eq para producir un $\mathrm{kg}$ de leche.

De acuerdo a los valores reportados en la literatura acerca del consumo de agua en la producción de leche, los valores obtenidos en este trabajo se encuentran por encima del rango mencionado por Chapagain \& Hoekstra (2003) y Mekkonen \& Hoekstra (2010), el cual variaba entre 760 y $1.800 \mathrm{~L}$ de agua por $L$ de leche. Al comparar los resultados obtenidos en este estudio, con los datos presentados por Sultana et al. (2014), se observa que el valor encontrado aquí, está dentro del rango definido por estos autores (739 - 5.622 $L$ de agua por $L$ de leche), pero por encima del promedio global $(1.833 \mathrm{~L})$ y del promedio para Suramérica (1.713 L).

Para la huella de carbono, el valor obtenido fue cercano a los 2,8 $\mathrm{kg} \mathrm{CO}$ eq / $\mathrm{kg}$ leche estimado por Gerber et al., (2013) como promedio global. Otros autores, siguiendo la metodología de las ecuaciones propuestas por el IPCC, como Rotz et al. (2010), Gerber et al. (2011) y Leschen et al. (2011), encontraron huellas de carbono entre $0,37-0,69 ; 1,6-1,8$ y 1,3 $\mathrm{kg} \mathrm{CO}$ eq / kg leche, respectivamente. Guerci et al. (2013), Serra (2013) y Thoma et al. (2013), reportaron valores entre 1,23 y $1,72 \mathrm{~kg} \mathrm{CO}$ eq/ $/ \mathrm{kg}$ leche. O'Brien et al. (2015), encontró huellas de carbono en lecherías irlandesas, bajo condiciones de pastoreo, entre 0,6 y $2,13 \mathrm{~kg} \mathrm{CO}{ }^{2} \mathrm{eq} / \mathrm{kg}$ leche.

Las diferencias encontradas tanto para la huella hídrica como la de carbono, con respecto a los autores mencionados anteriormente, podría deberse a la mayor producción diaria de leche por animal que reportan en estos estudios (> $10 \mathrm{~kg}$ leche/vaca/día) comparadas con los $5 \mathrm{~kg}$ producidos por el ganado doble propósito colombiano.

\section{Reducción en la edad al primer parto (EPP)}

Se redujo gradualmente el valor de la EPP hasta un valor biológicamente posibles para condiciones tropicales, desde el valor base de 36 meses, reportado como valor promedio nacional, hasta 30 meses. En la Tabla 5, se puede observar, como la reducción de un mes en la EPP, disminuía en promedio mensualmente, $123.156( \pm 6.874) \mathrm{L}$ de agua y $155 \mathrm{~kg}$ de $\mathrm{CO}_{2}$ eq. Para las huellas hídrica y de carbono, la reducción promedio fue de 20,94 L agua/L leche y $0,03 \mathrm{~kg} \mathrm{CO}$ eq $/ \mathrm{kg}$ leche, respectivamente.

Tabla 5. Efecto de la edad al primer parto (EPP) sobre el consumo de agua, GEl y las huellas de carbono e hídrica.

\begin{tabular}{c|c|c|c|c}
\hline $\begin{array}{c}\text { EPP } \\
(\text { meses })\end{array}$ & $\begin{array}{c}\text { Agua } \\
(\mathbf{L})\end{array}$ & $\begin{array}{c}\text { GEI } \\
(\mathbf{k g ~ C O} \text { eq) }\end{array}$ & $\begin{array}{c}\text { HH } \\
(\mathbf{L} \text { agua/L leche) }\end{array}$ & $\begin{array}{c}\text { HC } \\
(\mathbf{k g ~ C O} \text { eq/kg leche) }\end{array}$ \\
\hline 36 & 12.804 .569 & 16.066 & 2.178 & 2,73 \\
\hline 35 & 12.671 .815 & 15.911 & 2.155 & 2,71 \\
\hline 34 & 12.543 .102 & 15.755 & 2.133 & 2,68 \\
\hline 33 & 12.418 .430 & 15.600 & 2.112 & 2,65 \\
\hline 31 & 12.297 .799 & 15.445 & 2.092 & 2,63 \\
\hline 30 & 12.180 .199 & 15.290 & 2.072 & 2,6 \\
\hline
\end{tabular}

*EPP= Edad al primer parto, $\mathrm{GEI}=$ Gases de efecto invernadero; $\mathrm{HH}=$ Huella hídrica; $\mathrm{HC}=$ Huella de carbono. Las cantidades de agua y GEl son el total desde el nacimiento hasta el sacrificio. 
Adicionalmente, en la Figura 3, se puede observar como la reducción en la EPP, permitió disminuir la permanencia del animal en el hato, es decir, el bovino terminaba su cuarta lactancia con menor edad, pasando de 91 meses en la línea base a 85 meses para una EPP de 30 .

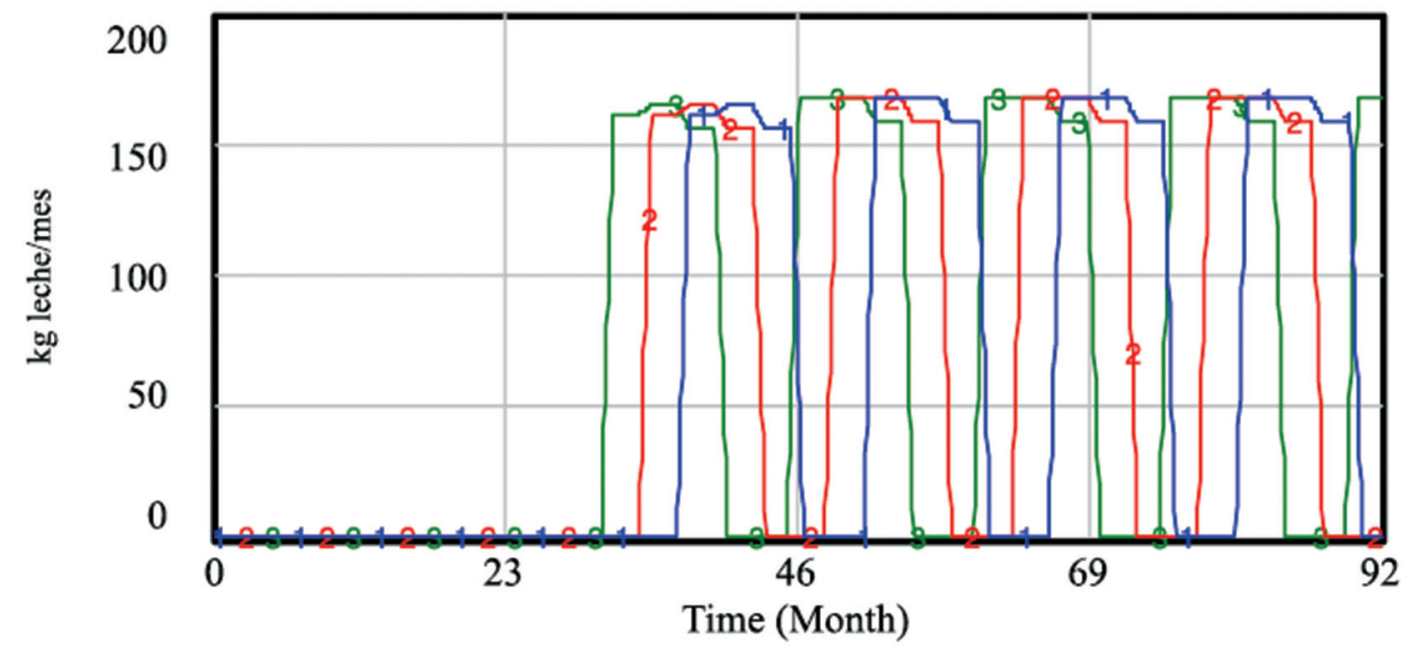

Lactancias y producción de leche : EPP 36 meses Lactancias y producción de leche : EPP 33 meses Lactancias y producción de leche : EPP 30 meses

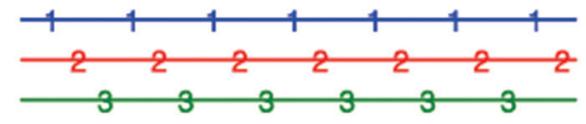

Figura 3. Efecto de la disminución en la edad al primer parto (EPP) sobre la permanencia del animal en el hato, cumplidas sus cuatro lactancias.

\section{Reducción en los días abiertos (DA)}

Para esta política, se redujo paulatinamente en 21 días (ciclo estral del bovino) el tiempo entre el parto y la concepción. En la Tabla 6, se puede observar como la reducción de un estro, disminuía en promedio mensualmente, 455.455 $( \pm 79.056) \mathrm{L}$ de agua y $647 \mathrm{~kg}$ de $\mathrm{CO}_{2}$ eq. Para las huellas hídrica y de carbono, la reducción fue de 93,21 L agua/L leche y $0,11 \mathrm{~kg} \mathrm{CO}$ eq/kg leche, respectivamente.

Tabla 6. Efecto de los días abiertos (DA) sobre el consumo de agua, GEl y las huellas de carbono e hídrica.

\begin{tabular}{c|c|c|c|c}
\hline DA (días) & $\begin{array}{c}\text { Agua } \\
(\mathbf{L})\end{array}$ & $\begin{array}{c}\text { GEI } \\
(\mathbf{k g ~ C O} \text { eq) }\end{array}$ & $\begin{array}{c}\text { HH } \\
\text { (L agua/L leche) }\end{array}$ & $\begin{array}{c}\text { HC } \\
\text { (kg CO } \mathbf{e q} / \mathbf{k g ~ l e c h e ) ~}\end{array}$ \\
\hline 171 & 12.804 .569 & 16.066 & 2.178 & 2,73 \\
150 & 12.163 .500 & 15.419 & 2.069 & 2,62 \\
\hline 129 & 11.824 .132 & 14.771 & 2.011 & 2,51 \\
\hline 87 & 11.317 .180 & 14.124 & 1.925 & 2,29 \\
\hline
\end{tabular}

*DA= Días abiertos, $\mathrm{GEI}=\mathrm{Gases}$ de efecto invernadero; $\mathrm{HH}=$ Huella hídrica; $\mathrm{HC}=$ Huella de carbono. Las cantidades de agua y GEI son el total desde el nacimiento hasta el sacrificio. 
En la Figura 4, se observa como la reducción en el intervalo entre el parto y la concepción, permitió disminuir la permanencia del animal en el hato, es decir, el bovino terminaba su cuarta lactancia con menor edad, pasando de 91 meses en la línea base a 79 meses para 66 días abiertos.

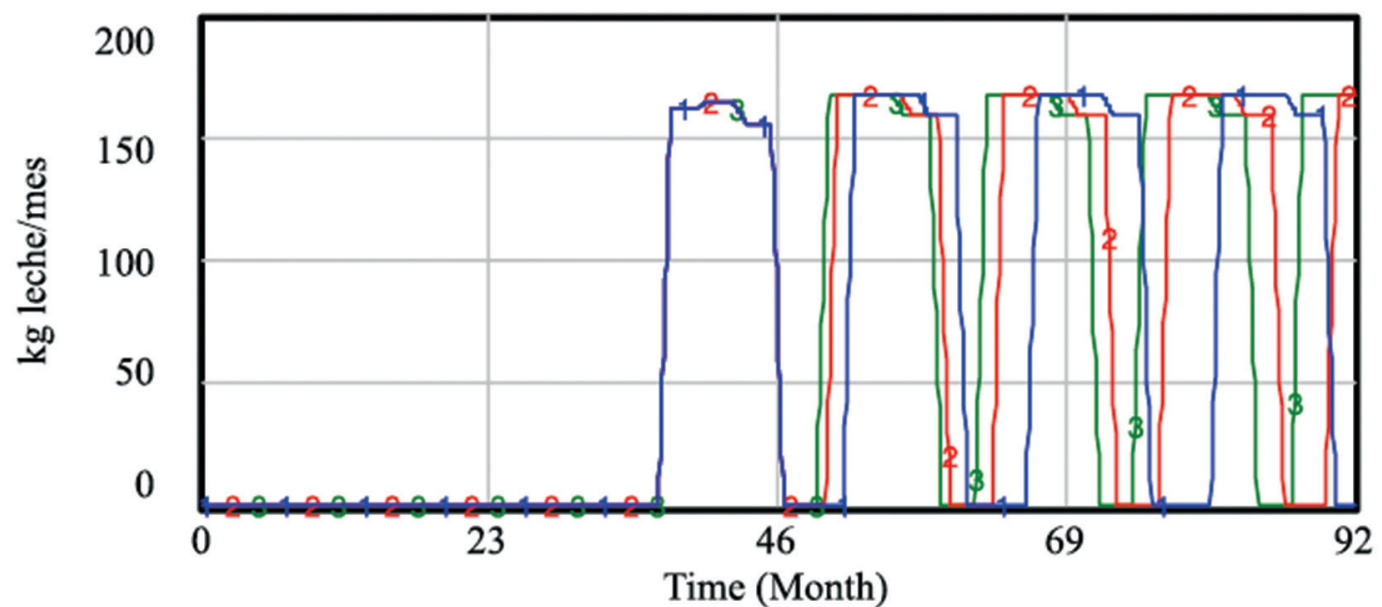

Lactancias y producción de leche : DA 171

Lactancias y producción de leche : DA 129

Lactancias y producción de leche : DA 66

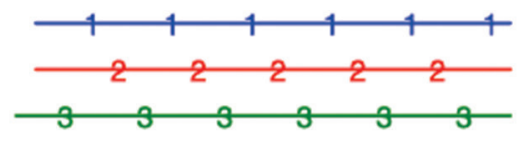

Figura 4. Efecto de la disminución en los días abiertos (DA) sobre la permanencia del animal en el hato, cumplidas sus cuatro lactancias.

La reducción en las cantidades de agua utilizada y GEI emitidos encontrados en este trabajo, a través de la disminución en los días abiertos y la edad al primer parto, ratifica las practicas de mitigación recomendadas por Hristov et al. (2013), a través de la crianza del ganado, en las cuales, la reducción en la edad para el sacrificio y la disminución de los días de alimentación, mediante mejoras en alimentación y genética, pueden tener un impacto significativo sobre las emisiones de GEl y uso de recursos naturales.

\section{Conclusiones}

Bajo el análisis puntual de esta investigación, se encontró que las modificaciones realizadas en aspectos reproductivos, edad al primer parto e intervalo entre el parto y la concepción, tienen una fuerte influencia sobre la dinámica del animal en el hato, producción de leche durante su vida, uso de recursos naturales y emisión de gases de efecto invernadero. Altos valores de estos parámetros afectarán la respuesta económica de la actividad ganadera, debido al menor crecimiento del hato y por consiguiente la baja rentabilidad de la actividad.

El modelo presentado en esta investigación proporciona una herramienta útil para académicos, investigadores y responsables del sector agropecuario del país, ya que permite conocer y comprender la complejidad de la actividad ganadera, proporcionando al tiempo instrumentos para la reflexión y la toma de decisiones, permitiendo actuar en diferentes escenarios, ensayar distintas políticas y experimentar las consecuencias de sus propias decisiones.

\section{Literatura citada}

1. Barragan, W., Mahecha, L. \& Cajas, Y. (2015). Variables fisiológicas-metabólicas de estrés calórico en vacas bajo silvopastoreo y pradera sin árboles. Agronomía Mesoam., 26(2):211-223.

2. Broussain, J. (2011). Análisis y aplicación de una metodología para el cálculo de la huella hídrica a nivel predial de la producción lechera en Chile. (Tesis de pregrado). Universidad de Chile. 
3. Chapagain, A. \& Hoekstra, A. (2003). Virtual water flows between nations in relation to trade in livestock and livestock products. Value of water research report series No. 13. UNESCO-IHE Institute for water education.

4. DANE. Cuentas Nacionales, Departamento Administrativo Nacional de Estadística DANE, (2011). Boletín mensual Insumos y factores asociados a la producción agropecuaria. Núm. 4 DANE.

5. FAO. (2009). The state of food and agriculture - livestock in the balance. Food and Agriculture Organization, Rome, Italy.

6. Fedegan. (2012). Foro de empresarización y competitividad ganadera: Costos y los indicadores de productividad en la ganadería colombiana. Recuperado de: http://www.slideshare.net/Fedegan/costose-indicadores-de-la-productividad-en-la-ganaderiacolombiana? related $=1$

7. Fedegan. (2013). Análisis del inventario ganadero colombiano: Comportamiento y variables explicativas. Bogotá, Colombia.

8. Gerber, P.; Vellinga, T., Opio, C. \& Steinfeld, H. (2011). Productivity gains and greenhouse gas emissions intensity in dairy systems. Livestock Science, 139; 100108.

9. Gerber, P.; Steinfeld, H., Henderson, B., Mottet, A., Opio, C., Dijkman, J., Falcucci, A. \& Tempio, G. (2013). Tackling climate change through livestock - A global assessment of emissions and mitigation opportunities. Food and Agriculture Organization of the United Nations (FAO), Rome.

10. Guerci, M., Bava, L., Zucali, M., Tamburini, A. \& Sandrucci, A. (2013). Effect of summer grazing on carbon footprint of milk in Italian Alps: A sensitivity approach. Journal of Cleaner Production, 73; 236-244.

11. Guimaraes, V.; Tedeschi, L. \& Teixeira, M. (2009). Development of a mathematical model to study the impacts of production and management policies on the herd dynamics and profitability of dairy goats. Agricultural system, 101, 186-196.

12. Herrero, M., Gerber, P., Vellinga, T., Garnett, T., Leip, A., Opio, C., Westhoek H,J., Thornton, P.K., Olesen, J., Hutchings, N., Montgomery, H., Soussana, J.F., Steinfeld, H. \& McAllister, T.A. (2011). Livestock and greenhouse gas emissions: the importance of getting the numbers right. Animal Feed Science and Technology 166-167, 779-782.

13. Herrero, M., Grace, D., Njuki, J., Johnson, N., Enahoro, D., Silvestri, S. \& Rufino, M. (2013). The roles of livestock in developing countries. Animal 7,3-18.

14. Hristov, A.N., Oh, J., Lee, C., Meinen, R., Montes, F., Ott, T., Firkins, J., Rotz, A., Dell, C., Adesogan, A., Yang, W., Tricarico, J., Kebreab, E., Waghorn, G., Dijkstra, J. \& Oosting, S. (2013). Mitigación de las emisiones de gases de efecto invernadero en la producción ganadera - Una revisión de las opciones técnicas para la reducción de las emisiones de gases diferentes al $\mathrm{CO}_{2}$. Editado por Pierre J. Gerber, Benjamin Henderson y Harinder P.S. Makkar. Producción y Sanidad Animal FAO Documento No. 177. FAO, Roma, Italia.
15. IDEAM - Instituto de Hidrología, Meteorología y Estudios Ambientales. Recuperado de: www.ideam.gov.co

16. IDF (International Dairy Federation). (2010). Environmental issues at dairy farm level. Bull. Int. Dairy Fed. Ser. No. 443, 3.

17. IPCC. (2006a). Chapter 10. Emissions from Livestock and Manure Management. In 2006 IPCC Guidelines for National Greenhouse Gas Inventories. Agriculture, Forestry and Other Land Use. Volume 4: 10.1-10.87.

18. IPCC. (2006b). Chapter 11: N2O Emissions from Managed Soils, and CO2 Emissions from Lime and Urea Application. In 2006 IPCC Guidelines for National Greenhouse Gas Inventories. Agriculture, Forestry and Other Land Use. Volume 4, 11.1-11.54.

19. IPCC. (2007). Intergovernmental Panel on Climate Change. Climate change 2007: The physical science basis. Contribution of Working Group I to the Fourth Assessment Report of the Intergovernmental Panel on Climate Change. Chapter 2: Changes in atmospheric constituents and in radiative forcing. Recuperado de: www.ipcc.ch/pdf/assessment-report/ar4/wg1/ar4-wg1chapter2.pdf

20. Key, N, Stacy S. \& David. M. (2014). Climate Change, Heat Stress, and U.S. Dairy Production, ERR-175, U.S. Department of Agriculture, Economic Research Service, September 2014.

21. Large Ruminal Nutrition System (LRNS) software Vensim PLE Plus (Ventana System, Inc.), Recuperado de: http://nutritionmodels.com/lrns.html

22. Lascano, C.; Ayarza, M., Cárdenas, E., Carulla, J., Boshell, F. \& Peña, A. (2009). Ante el cambio climático: Los desafíos de la producción ganadera. Ciencia y tecnología ganadera, 68. p. 4.

23. Lesschen, J.P., van den Berg, M., Westhoek, H.J., Witzkec, H.P. \& Oenema, O. (2011). Greenhouse gas emission profiles of European livestock sectors. Animal Feed Science and Technology, 166-167; 16-28.

24. Mekonnen, M. M. \& Hoekstra, A.Y. (2010). The green, blue and grey water footprint of farm animals and animal products. Value of Water Res. Rep. Ser. No. 48. UNESCO-IHE, Delft, the Netherlands.

25. Mekonnen, M.M. \& Hoekstra, A.Y., (2012). A global assessment of the water footprint of farm animal products. Ecosystems, 15, 401- 415.

26. Meyer, U., Everinghoff, D., Gadeken and G. \& Flachowsky, G. (2004). Investigations on the water intake of lactating dairy cows. Liv. Prod. Sci. 90:117-121.

27. Molina, R. (2015). Estudio de la sostenibilidad en cuatro sistemas ganaderos con diferentes niveles de intensificación mediante dinámica de sistemas. Tesis de doctorado. Universidad Nacional de Colombia. p. 162.

28. Molina, R., Sánchez, H. \& Atzori, A. (2015). Dynamic stimation of the carbon footprint for a dual purpose cattle. XIII congreso latinoamericano y encuentro colombiano de dinámica de sistemas. Colombia, Octubre de 2015. p. 51-57.

29. NAP. National Academies Press. (2011). Sustainability and the U.S. EPA. Washington, D.C. p. 150. 
30. O’Brien, D., Hennessy, T., Moran, B. \& Shalloo, L. (2015). Relating the carbón footprint of milk from Irish dairy farms to economic performance. Journal of Dairy Science, 98: 7394-7407.

31. Palmett, L. (2015). El impacto del posconflicto en el sector agrario colombiano, un análisis desde la gestion pública. Universidad Militar Nueva Granada.

32. Pereira, V. (2007). Modelagem de uma unidade de produção para caprinos leiteiros utilizando a dinâmica de sistemas. Doctor Scientiae tese. Universidade Federal de Vicosa.

33. Perfetti, J., Balcázar, A., Hernández, A., Leibovich, J., Becerra, A., Botello, S., Cortés, S., Estrada, L., Rodríguez, C. \& Vásquez, H. (2013). Políticas para el desarrollo de la agricultura en Colombia. SAC y FedesarroIlo. Bogotá D.C. p 247

34. Peters, M.; Franco. L. H., Schmidt, A. \& Hincapié, B. (2011). Especies forrajeras multipropósito: Opciones para productores del trópico americano. Publicación CIAT 374. p. 212.

35. PNUD. (2011). Colombia rural. Razones para la esperanza. Informe Nacional de Desarrollo Humano 2011. Bogotá: INDH PNUD, septiembre. p. 443.

36. Rotz, C., Montes, F. \& Chianese D. (2010). The carbon footprint of dairy production systems through partial life cycle assessment. Journal of Dairy Science, 93, 1266-1282.

37. Schlink, A., Nguyen, M. \& Viljoen, G. (2010). Water requirements for livestock production: a global perspective. Rev. sci. tech. Off. int. Epiz., 2010, 29 (3), 603-619.

38. Senge, P. (1990). La Quinta Disciplina. Granica. p. 75.
39. Serra, M. (2013). Estimation of carbon footprint in dairy cattle farms of Southern Italy. PhD thesis. Università degli Studi di Sassari. Italy.

40. Sterman, J.D. (2000). Business Dynamics, Systems Thinking and Modeling for a Complex World; McGrawHill: Boston, MA, USA.

41. Stockdale, C. R. \& K. R. King. (1983). A note on some of the factors that affect the water consumption of lactating dairy cows at pasture. Anim. Prod. 36:303-306.

42. Sultana, Mst.N., et al. (2014). Benchmarking consumptive water use of bovine milk production systems for 60 geographical regions: An implication for Global Food Security. Global Food Security, http://dx.doi. org/10.1016/j.gfs.2014.08.006i

43. Thoma, G., Popp J., Nutter D., Shonnard D., Nutter, D., Matlock M., Ulrich R., Kellogg, W., Kim D.S., Neiderman Z., Kemper N., Adom, F. \& East C. (2013). Regional analysis of Greenhouse gas emissions from US dairy farms: a cradle-to- farm-gate assessment of the American dairy industry circa 2008. International Dairy Journal 31; S29-S40.

44. Thornthwaite, C.W. (1948). An approach toward a rational classification of climate. Geograph. Rev., 38, 55.

45. Winchester, C. F., and M. J. Morris. (1956). Water intake rates of cattle. J.Anim. Sci. 15:722-740.

46. World Bank (2009). Awakening Africa's sleeping giant: prospects for commercial agriculture in the Guinea savannah zone and beyond World Bank, Washington, DC. Recuperado de: http://siteresources.worldbank. org/ INTARD/Resources/sleeping_giant.pdf
Conflicto de Intereses

Los autores declaran no tener ningún conflicto de intereses

Recibido: 22 de marzo de 2016 Aceptado: 03 de mayo de 2016 